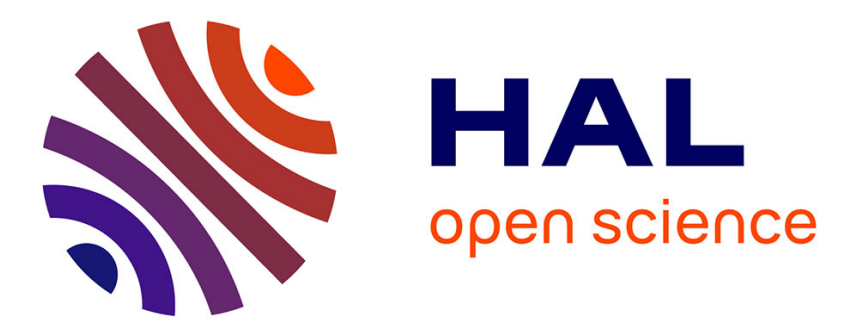

\title{
Molecules as Diagnostic Tools in the Interstellar Medium
}

Nicole Feautrier, Annie Spielfiedel, Christian Balança, Fabrice Dayou, Francois Lique, Maria Luisa Senent

\section{To cite this version:}

Nicole Feautrier, Annie Spielfiedel, Christian Balança, Fabrice Dayou, Francois Lique, et al.. Molecules as Diagnostic Tools in the Interstellar Medium. Molecular Physics, 2007, 105 (09), pp.1263-1269. 10.1080/00268970701361306 . hal-00513096

\section{HAL Id: hal-00513096 https://hal.science/hal-00513096}

Submitted on 1 Sep 2010

HAL is a multi-disciplinary open access archive for the deposit and dissemination of scientific research documents, whether they are published or not. The documents may come from teaching and research institutions in France or abroad, or from public or private research centers.
L'archive ouverte pluridisciplinaire HAL, est destinée au dépôt et à la diffusion de documents scientifiques de niveau recherche, publiés ou non, émanant des établissements d'enseignement et de recherche français ou étrangers, des laboratoires publics ou privés. 


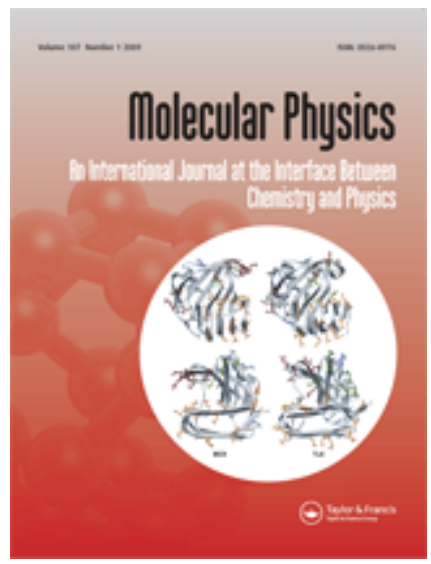

Molecules as Diagnostic Tools in the Interstellar Medium

\begin{tabular}{|c|c|}
\hline Journal: & Molecular Physics \\
\hline Manuscript ID: & TMPH-2006-0114.R1 \\
\hline Manuscript Type: & Full Paper \\
\hline $\begin{array}{r}\text { Date Submitted by the } \\
\text { Author: }\end{array}$ & 16-Mar-2007 \\
\hline Complete List of Authors: & $\begin{array}{l}\text { Feautrier, Nicole; Observatoire de Paris, LERMA } \\
\text { Spielfiedel, Annie; Observatoire de Paris, LERMA } \\
\text { Balanca, Christian; Observatoire de Paris, LERMA } \\
\text { Dayou, Fabrice; Observatoire de Paris, LERMA } \\
\text { Lique, Francois; Observatoire de Paris, LERMA } \\
\text { Senent, Maria Luisa; IEM-CSIC, DAMIR }\end{array}$ \\
\hline Keywords: & $\begin{array}{l}\text { Potential energy surface, inelastic collision, rotational } \\
\text { excitation, interstellar medium }\end{array}$ \\
\hline \multicolumn{2}{|c|}{$\begin{array}{l}\text { Note: The following files were submitted by the author for peer review, but cannot be converted } \\
\text { to PDF. You must view these files (e.g. movies) online. }\end{array}$} \\
\hline Spielfiedel.tex & \\
\hline
\end{tabular}

\section{S) ScholaroNE \\ Manuscript Central}


Molecular Physics, Vol. 00, No. 00, DD Month 200x, 1-8

\title{
Molecules as Diagnostic Tools in the Interstellar Medium
}

\author{
A. SPIELFIEDEL $\dagger$, N. FEAUTRIER ${ }^{*} \dagger$, C. BALANÇA $\dagger$, F. DAYOU ${ }^{* *} \dagger$, F. LIQUE*** $\dagger$ and M.-L. SENENT $\ddagger$ \\ †LERMA, UMR 8112 du CNRS, 5 Place Jules Janssen, 92195 Meudon CEDEX, France \\ †DAMIR. IEM-CSIC. C/Serrano 121. 28006 Madrid. Spain \\ (Received)
}

\begin{abstract}
Analysis of light emission from different regions of the interstellar medium and circumstellar environments provides crucial information about the chemical composition and the physical conditions in these regions. Interpretation of the observed spectra requires the knowledge of collisional excitation rates as well as radiative rates participating to the line formation. In a first part, the paper focuses on collisional excitation rates of molecules relevant to the interstellar medium. It discusses currently available data and outlines new work carried out by the authors. Due to the use of accurate ab initio potential energy surfaces, the new rate coefficients differ significantly from previously published ones. In a second part, it is analysed from two examples how the use of the new rate coefficients could lead to important
\end{abstract} changes in the interpretation of molecular emission emerging from molecular clouds.

Keywords: Potential energy surface, inelastic collision, rotational excitation, interstellar medium,

\section{Introduction}

Over the next few years, ground-based and space-based missions will open up the universe to high spatial and spectral resolution studies at infrared and submillimeter wavelengths. This will allow to study, in much greater details, the molecular composition of a variety of astrophysical regions, and, subsequently, to trace the origin and evolution of molecules in space. These new missions are expected to lead to the detection of many thousands of new spectral features. Identification, analysis and interpretation of these features, in terms of the physical and chemical characteristics of the astronomical sources, will require detailed astronomical tools supported by both laboratory measurements and theoretical calculations of spectroscopical data, reaction rate coefficients and collisional excitation rates for species of astrophysical relevance.

Whereas a proper modeling of interstellar molecular emission requires excitation calculations using collisional rate coefficients, to date, the determination of such data by means of the most accurate methods concern only few molecular systems of astrophysical interest. The collisional rate coefficients with the most abundant interstellar species $\mathrm{He}$ and $\mathrm{H}_{2}$ as colliders are needed given that the steady-state populations in each ro-vibrational level of molecules are driven by the competition between collisional and radiative processes. Taking into account the sensitivity of the radiative transfer models to the collisional rate coefficients, and the influence of careful calculation of the excitation rate coefficients on the interpretation of observed spectra $(1 ; 2 ; 3)$, it is thus of a great importance to provide accurate values of these rate coefficients for the molecules of astrophysical interest.

The present paper focuses in a first part on recent theoretical studies of collisional excitation processes at low temperatures for several molecular systems with $\mathrm{He}$ as a collider. In cold environments such as

\footnotetext{
* Corresponding author. Email: Nicole.Feautrier@obspm.fr

** Present adress: Departamento de Astrofisica Molecular e Infrarojo, IEM-CSIC. C/Serrano 121. 28006 Madrid. Spain

*** Present adress: Department of Chemistry and Biochemistry, University of Maryland, College park, Maryland 20742-2021, USA

Molecular Physics

ISSN 0026-8976 print/ ISSN 1362-3028 online (C)2005 Taylor \& Francis Ltd

http://www.tandf.co.uk/journals

DOI: $10.1080 / 002689700 \times x \times x x x \times x x x x x$
} 
the interstellar medium (ISM), collisions with $\mathrm{H}_{2}$ are the most important due to its high abundance. Nonetheless, because of the near-sphericity of $\mathrm{H}_{2}$ and its large rotational constant, collisions with He can be considered as a model for collisions with para- $\mathrm{H}_{2}$. In a second part of the paper, we make use of the newly obtained rate coefficients to analyse their impact on astrophysical modeling through radiative transfer models.

\section{Molecular Collisions in the Interstellar Medium}

\subsection{Critical densities}

The steady-state population in each quantum state of a molecular system depends on the competition between radiative and collisional processes. Their determination in the optically thin approximation relies on the statistical equilibrium equations(4), written for each level $j$ as:

$$
\begin{aligned}
0= & \sum_{l>j}\left[n_{l} A_{l j}+\left(n_{l} B_{l j}-n_{j} B_{j l}\right) J\right] \\
& -\sum_{l<j}\left[n_{j} A_{j l}+\left(n_{j} B_{j l}-n_{l} B_{l j}\right) J\right]+\sum_{l} n_{P}\left[n_{l} k_{l j}-n_{j} k_{j l}\right]
\end{aligned}
$$

where the A and B terms are the Einstein coefficients for spontaneous and stimulated emission and absorption associated with the quantum states $l$ and $j$, for the incident radiative field $J$. The $k_{j l}\left(k_{l j}\right)$ terms are the collision rate coefficients from $j$ to $l(l$ to $j)$ and $n_{P}$ is the density of colliding particles $\left(\mathrm{H}_{2}\right.$ or $\left.\mathrm{He}\right)$. The collision rate coefficients at a temperature $T$ are related to the collisional cross sections through:

$$
k_{i j}(T)=\left(\frac{8 k_{B} T}{\pi \mu}\right)^{\frac{1}{2}}\left(\frac{1}{k_{B} T}\right)^{2} \times \int_{0}^{\infty} E_{k} \sigma_{i j}\left(E_{k}\right) \exp \left(-E_{k} / k_{B} T\right) d E_{k}
$$

The knowledge of the collision rate coefficients allows one to define for each level $j$ a critical density $n_{j}^{c r i t}$ such that (5):

$$
n_{j}^{c r i t}=\frac{A_{j \rightarrow j-1}}{\sum_{i} k_{j i}}
$$

where the sum runs over all reachable states. We may consider that the so-called Local Thermodynamical Equilibrium is fulfilled if the density $n_{P}$ is much larger than $n_{j}^{\text {crit }}$, since collisions tend to equilibrate the populations of the considered species in such a case. Conversely, the radiative processes are dominant for densities much lower than $n_{j}^{\text {crit }}$. Between these two extreme cases, both processes compete and accurate collision rates with the most abundant species are thus needed for the precise determination of the steadystate populations, and subsequently the physical conditions of the astronomical source. It is clear that studying different molecules and different ro-vibrational levels of each of them allows one to explore different regions of the studied astrophysical object. This point will be discussed in more details in the last section.

\subsection{Potential energy surfaces}

Within the Born Oppenheimer approximation, the determination of the collision rate coefficients requires two steps: the calculation of the interaction between nuclei and electrons for fixed nuclear geometries, leading to the so-called potential energy surface (PES), and the determination of the scattering cross 
sections associated with the molecular fragments for each quantum state of interest. In the context of observations in the $\mathrm{mm}$ and submm ranges, the collisional processes to be studied concern astrophysical regions characterized by relatively low temperatures $(5 \mathrm{~K}<T<1500 \mathrm{~K})$. Moreover, for $\mathrm{H}_{2}$ or $\mathrm{He}_{\mathrm{s}}$ the colliding particles, the molecular systems considered are generally of a van der Waals type. Under such conditions, electronic excitations and reactive channels are quite unlikely, and the collisional process can be treated by means of a single PES.

Recent progress in ab initio quantum chemistry facilitate very accurate determinations of the PES of small non-reactive systems. For such molecular systems, the coupled cluster theory including singles, doubles and perturbative triples excitations $(\mathrm{CCSD}(\mathrm{T}))$ is generally assumed to be very accurate, as long as only one configuration contributes significantly to the electronic state. It is generally the case for collisional excitation of rotational states in the interstellar medium, given that the temperature is very low and the relevant species are in their ground vibrational state. In that case, the geometries of the two partners are frozen at their equilibrium values, or their average values within the ground vibrational state. For collisions at higher energies, leading to possible vibrational excitation, this point must be checked out carefully as more than one configuration may contribute to the ground electronic PES when the interatomic distances of the studied molecule vary. In the case that a single electronic configuration is no more fulfilled, multiconfigurational approaches, such as the CASSCF-MRCI, may thus be needed to properly describe the PES.

In the following we discuss the results obtained for the CS, SiO and SO molecules with He as the collider. For each of these van der Waals systems, the determination of the PES relied on the use of the coupled-cluster method at the CCSD(T) level of theory. We employed the augmented correlation-consistent atomic basis sets of quadruple zeta quality (aug-cc-pVQZ)(7). To improve further the description of the wavefunction between the interacting fragments, we have added to this set the (3s $3 \mathrm{p} 2 \mathrm{~d} 2 \mathrm{f} 1 \mathrm{~g})$ bond functions optimized by Cybulski and Toczylowski(8), placed at the midpoint betwen the He atom and the studied diatom. The calculated interaction energies were corrected from the basis set superposition error (BSSE) at all geometries using the Boys and Bernardi(6) counterpoise procedure. All the calculations were performed with the MOLPRO suite of programs(9).

\subsection{Dynamics}

The most reliable approach to compute inelastic scattering cross sections is to perform quantum calculations by mean of the close-coupling method, first introduced by Arthurs \& Dalgarno(10). The main limitation of such a theoretical approach comes from the large number of coupled channels that increases rapidly with energy, in relation with the number of available levels to be accounted for. While the exact close coupling approach, including all the angular momentum couplings, is feasible for rotational excitation of small molecules at low energies, it becomes unrealistic if the number of channels becomes too large, particularly if vibrational excitation has to be included. There exist various approximations (quantal or semi-classical) to the full close-coupling approach which were developed to circumvent such a limitation. Among them, the fast and popular Infinite Order Sudden (IOS) quantum approximation usually converges well whenever the relevant $\Delta j$ rotational energy spacings are small compared to the available collisional energy. When the energy is increased this condition is fulfilled for the lower $j$ levels, but not for the highest $j$ levels. In that respect, the extension of the present scattering calculations to the obtention of inelastic rates for warm gas or shocked regions may be problematic if accurate results are sought. All the results presented here were obtained within the full close-coupling approach using the MOLSCAT code(11).

\subsection{Ro-vibrational excitation of interstellar species by $\mathrm{He}$}

2.4.1 $\boldsymbol{C S}$ and $\boldsymbol{S i O}$. For these two molecules, the rigid rotor PESs were described by the two Jacobi coordinates $[1 ; 12]: R$ (distance from the center of mass of the diatom to the He atom) and $\theta$ (angle between $\mathbf{R}$ and the diatomic bond axis), the r-distances between the $\mathrm{C}-\mathrm{S}$ and $\mathrm{Si}-\mathrm{O}$ atoms were frozen at their experimental equilibrium values $\left(r_{e}=2.90\right.$ bohr for $\mathrm{CS}$ and $2.853 \mathrm{bohr}$ for $\left.\mathrm{SiO}\right)$. $\theta=0$ corresponds to He adjacent to the $\mathrm{C}$ atom for the CS-He PES and to the $\mathrm{O}$ atom for the SiO-He PES. In both calculations, 
the angular grid varied from 0 to 180 degrees by steps of 15 degrees. For the CS-He surface, the radial coordinate $R$ was assigned values from $3.5 \mathrm{bohr}$ to $16.0 \mathrm{bohr}$ by steps of $0.25 \mathrm{bohr}$. The global minimum of the CS-He interaction energy was found to be $-21.8051 \mathrm{~cm}^{-1}(\mathrm{R}=7.26 \mathrm{bohr}, \theta=75$ degree). For the SiOHe surface, $R$ ranged from 4.0 to 16.0 bohr by steps of 0.25 bohr and, at short atom-diatom separations, additional geometries were computed to insure that the interaction energy becomes at least $3500 \mathrm{~cm}^{-1}$ at any angle. The global minimum of the SiO-He interaction energy was found to be $-26.596 \mathrm{~cm}^{-1}$ at the linear He-OSi geometry $(R=7.993 \mathrm{bohr})$. The calculated interaction energies were fitted by means of the procedure described by Werner et al. (1988) in order to obtain the $V\left(r=r_{e}, R, \theta\right)$ numerical expansion required for scattering calculations.

The new CS-He and SiO-He ab initio PESs were employed to perform full close-coupling calculations of the rotational (de)excitation cross sections for energies up to $3000 \mathrm{~cm}^{-1}(1 ; 12)$. The leading rotational de-excitation cross sections are decreasing functions of increasing kinetic energies, and display oscillations at low collision energies corresponding with resonances (of shape or Feshbach type) due to the presence of an attractive van der Waals potential well in the PES. Thermal average of the scattering cross sections yields the collision rate coefficients. We report in Fig. 1 the de-excitation rate coefficients of both CS and $\mathrm{SiO}$ molecules for a given initial rotational level $j=7$ as a function of $\Delta j=j-j^{\prime}$. It is seen that the de-excitation rate coefficients decrease with increasing $\Delta j$, and propensity rules arise, namely: even $\Delta j$ rate coefficients larger than odd $\Delta j$ ones for CS and the reverse for $\mathrm{SiO}$. Such behaviour is observed for all initial $j$ level and all temperatures. Whereas the magnitude of the inelastic collision rate coefficients is driven by the anisotropy of the PES, almost similar for both molecular systems, the opposite propensity rules observed relate with the asymmetric character of each PES(14). In the case of CS-He collisions, the PES displays a shallow potential well of about $22 \mathrm{~cm}^{-1}$ close to a T-shape orientation of the fragments. Under such conditions, propensity rules almost similar to that of symmetric molecules are obtained (the excitation corresponding with odd $\Delta j$ transitions is forbidden in such a case). Conversely, for the SiO-He PES a shallow potential well of about $27 \mathrm{~cm}^{-1}$ is also obtained but at the linear He-OSi geometry, mainly due to the large differences between the atomic polarizabilities of the $\mathrm{Si}$ and $\mathrm{O}$ atoms $\left(\alpha_{\mathrm{Si}} \gg \alpha_{\mathrm{O}}\right)$, that strongly favour odd $\Delta j$ transitions over even $\Delta j$ ones.

The rate coefficients for collisions with para- $\mathrm{H}_{2}(j=0)$ are expected to be larger than those associated with $\mathrm{He}$ as a collider, owing to the smaller reduced mass of the two collision partners in the case of para- $\mathrm{H}_{2}$. It is thus often applied a scaling factor to the collision rate coefficients with He to get a first estimate of the rates with para- $\mathrm{H}_{2}$, the scaling factor being close to 1.4 for molecules much heavier than $\mathrm{H}_{2}$. In such a case the underlying approximation is to consider identical cross section values for the two colliding systems. Another widely used approximation is to consider the interaction potential between a given molecule and He identical to the one associated with para- $\mathrm{H}_{2}$, and then perform scattering calculations using the reduced mass corresponding with para- $\mathrm{H}_{2}$ to get the associated rate coefficients. The previous results for 
the CS-para $\mathrm{H}_{2}$ and $\mathrm{SiO}$-para $\mathrm{H}_{2}$ colliding systems were estimated in such a way by Turner et al.(15), using CS-He and SiO-He PESs derived from the electron gas model. To assess the effect of the new ab initio PES on collision rate coefficients, we employed an identical approximation. For the two colliding systems, the comparison with the results of Turner(15) shows quite important disagreements, from a factor 2 up to 5 depending on the temperature and $\Delta j$, that clearly point out the major role played by the PES.

2.4.2 SO. The previously available rate coefficients were provided by Green(16) for the $\mathrm{SO}-\mathrm{H}_{2}$ system at temperatures ranging from $50 \mathrm{~K}$ to $350 \mathrm{~K}$. The author used a $\mathrm{CS}-\mathrm{H}_{2} \mathrm{PES}$ adapted from an electron gas model of CS-He. The rate coefficients among fine levels were obtained with a IOS scaling relationship(17). A new ab-initio PES was calculated using the CCSD(T) method and the basis sets described in paragraph 2.2. The SO r-distance was frozen at its experimental minimum energy distance $\left(r_{e}=2.80 \mathrm{bohr}\right)$. The values of the radial coordinate $R$ ranged from 4 bohr to 16 bohr in steps of 0.25 bohr. The angular grid was uniform with a 15 degree spacing from 0 to 180 degree. At some angles, it was necessary to include smaller values of $R$ to ensure that the calculations extended into the short-range repulsive region of the potential. The global minimum was found to be $-34.921 \mathrm{~cm}^{-1}(\mathrm{R}=7.29 \mathrm{bohr}, \theta=180$ degrees $)$. The fitting procedure of Werner et al. (1988) was used.

From the new ab-initio PES, we have carried out full close coupling calculations with the exact energy levels including the fine structure interaction. Rate coefficients were obtained for temperatures ranging from $5 \mathrm{~K}$ to $50 \mathrm{~K}(18)$. The results displayed the expected propensity rule $\Delta j=\Delta N$.

The use of recoupling techniques from spin-free cross sections was investigated at low energy. This approximation is not valid for excitation between the first levels $(N \leq 5)$ where the fine structure splitting is large compared to the rotational splitting but it provides a reasonable estimate of rates for high-J levels

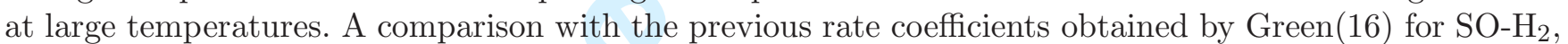
and transformed into SO-He coefficients by the ratio of masses, are on average a factor of 2-3 below the recent ones for the $\Delta j=\Delta N$ transitions. As previously discussed for CS-He and SiO-He collisions, the precision of the PES appears to be also critical for the SO-He system. However, the differences may be as large as a factor of 10 for transitions involving the low-energy levels, in particular for the $0_{1}-1_{0}$ transition. This is due to the fine structure interaction (not explicitly included by Green) which leads to the $1_{0}$ level energetically lower than the $0_{1}$ level. We thus consider that the new collision rate coefficients are more reliable than the previous ones.

\section{Astrophysical consequences}

The population of the ro-vibrational levels of interstellar molecules is determined by both the density and by the radiation field (statistical equilibrium equations) whereas the radiation field is also determined by the population of the ro-vibrational levels of interstellar molecules. Indeed, we use radiative transfer codes to solve simultaneously the statistical equilibrium equations and the radiative transfer equation. These codes allow the determination of the physical conditions of molecular clouds (temperature, density, abundance of molecule ...) since, using them, we can simulate, for a set of physical conditions of a molecular cloud, the spectral line shapes of ro-vibrational transitions of observed molecules and compare them to the observations in order to validate the adopted model of the cloud.

The consequence on the determination of ISM physical conditions of using one set of collision rate coefficients or another is direct as the rate coefficients contribute through the product $k_{i j} n_{P}$ to the statistical equilibrium equations (equation 1 ): increasing the rate coefficients $k_{i j}$ is equivalent to decreasing the density $n_{P}$.

Taking as example the SO molecule, we have studied the astrophysical effect of the new rates by modeling the excitation of SO through a Large Velocity Gradient (LVG) radiative transfer code(19). We have compared(2) the intensities $I_{\nu}$ (expressed in terms of brightness temperature $\left.T_{B}=\left(c^{2} / 2 k \nu^{2}\right) I_{\nu}\right)$ obtained for observed $0_{1}-1_{0}, 2_{2}-1_{1}, 2_{3}-1_{2}, 6_{5}-5_{4}$ lines by using the rates of Lique et al.(18) and those of Green(16). The corresponding ratio is plotted in Fig. 2 as a function of the density for SO column densities ranging from $3.10^{12}$ to $3.10^{15} \mathrm{~cm}^{-2}$ by step factors of 10 . 

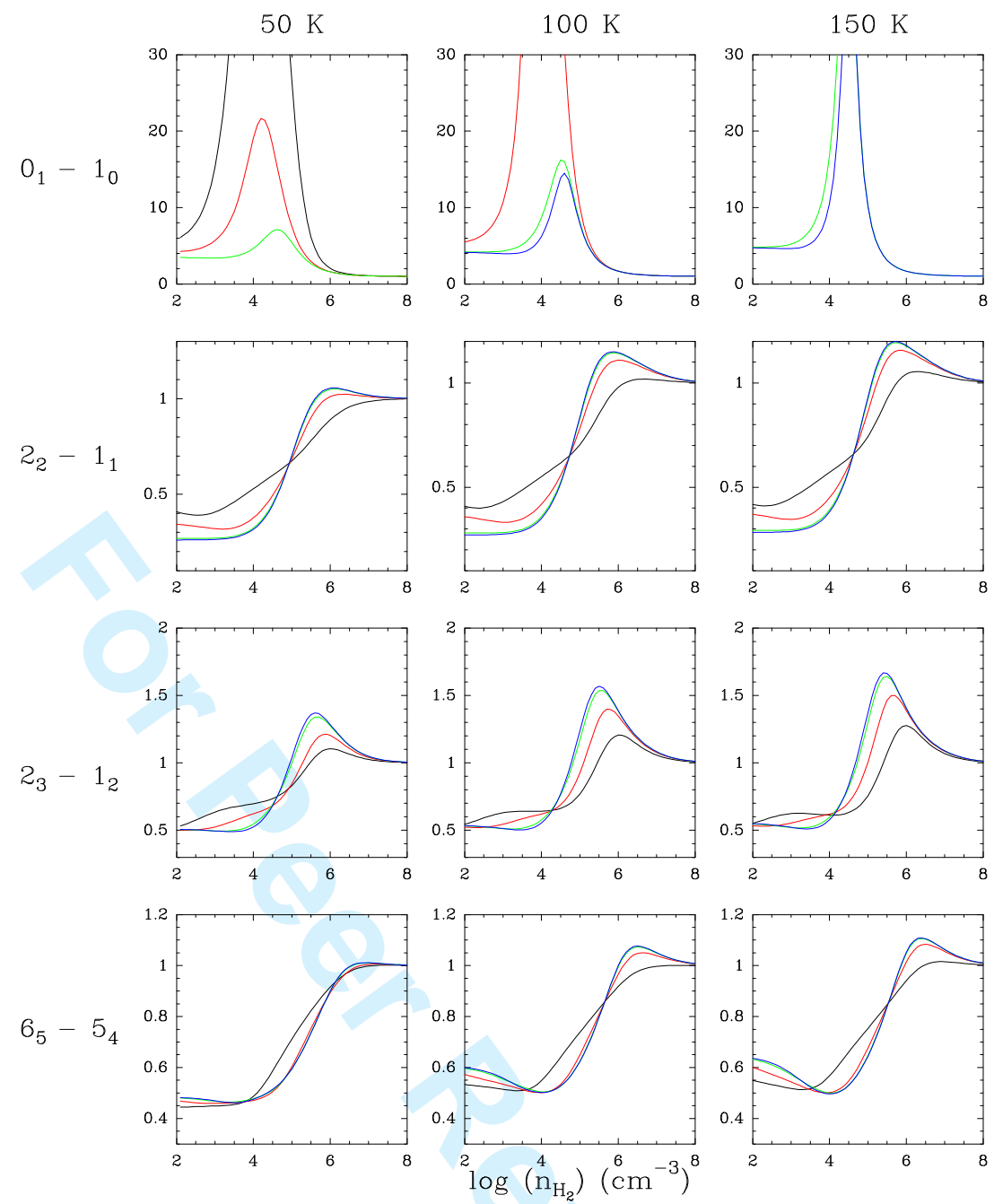

Figure 2. Brightness temperature ratios $\left(\mathrm{T}_{B}\right.$ (Green [13] rate coefficients) $/ \mathrm{T}_{B}\left(\right.$ Lique et al. [15])) for $0_{1}-1_{0}, 2_{2}-1_{1}, 2_{3}-1_{2}$ and $6_{5}-5_{4}$ lines of SO (from top to bottom). The $\mathrm{H}_{2}$ volume density varies between $10^{2}$ and $10^{8} \mathrm{~cm}^{-3}$ and the $\mathrm{SO}$ column density from 3 $10^{12}$ and $310^{15} \mathrm{~cm}^{-2}$ by a step factor of 10 (blue, green, red and black lines, respectively). From left to right the kinetic temperature corresponds to 50,100 , and $150 \mathrm{~K}$, respectively.

We can see that the brightness temperature ratio is really different from one and that differences are important especially for the frequently observed $0_{1}-1_{0}$ line. In addition, the ratio is larger than one for the $0_{1}-1_{0}$ line whereas the situation is opposite for the other lines. The derivation of density or abundance of SO from observations will hence be dependent of the transitions used if the rate coefficients of Green(16) are used : if the $0_{1}-1_{0}$ line is used, the derived abundance of SO (or density) will be underestimated whereas an overestimation of the abundance of SO (or density) will be found if the other transitions are used.

We have applied these results to modeling of observations in TMC-1. We have used the observed line profiles of the transitions $0_{1}-1_{0}, 2_{3}-1_{2}, 3_{4}-2_{3}$ and $2_{2}-1_{1}$ in order to derive physical conditions and the $\mathrm{SO}$ abundance in this molecular cloud. We have used a non-local radiative transfer code and by comparing observations and synthetic spectra, we have built a model which allows a good fit of the observed spectra as shown in Fig. 3 (see (2) for details).

The structure of TMC-1 is well described by a cylindric filament containing several cores $\left(\mathrm{T}_{K}=8 \mathrm{~K}\right.$, $\left.\mathrm{n}\left(\mathrm{H}_{2}\right) \sim 310^{4} \mathrm{~cm}^{-3}\right)$ along the ridge and an envelope $\left(\mathrm{T}_{K}=10 \mathrm{~K}, \mathrm{n}\left(\mathrm{H}_{2}\right) \sim 6-810^{3} \mathrm{~cm}^{-3}\right)$ surrounding these condensations.

Compared to previously published determinations of the SO abundance in this molecular cloud using Green's rate coefficients(16), the new results on SO appear to be more robust, providing better diagnostics 

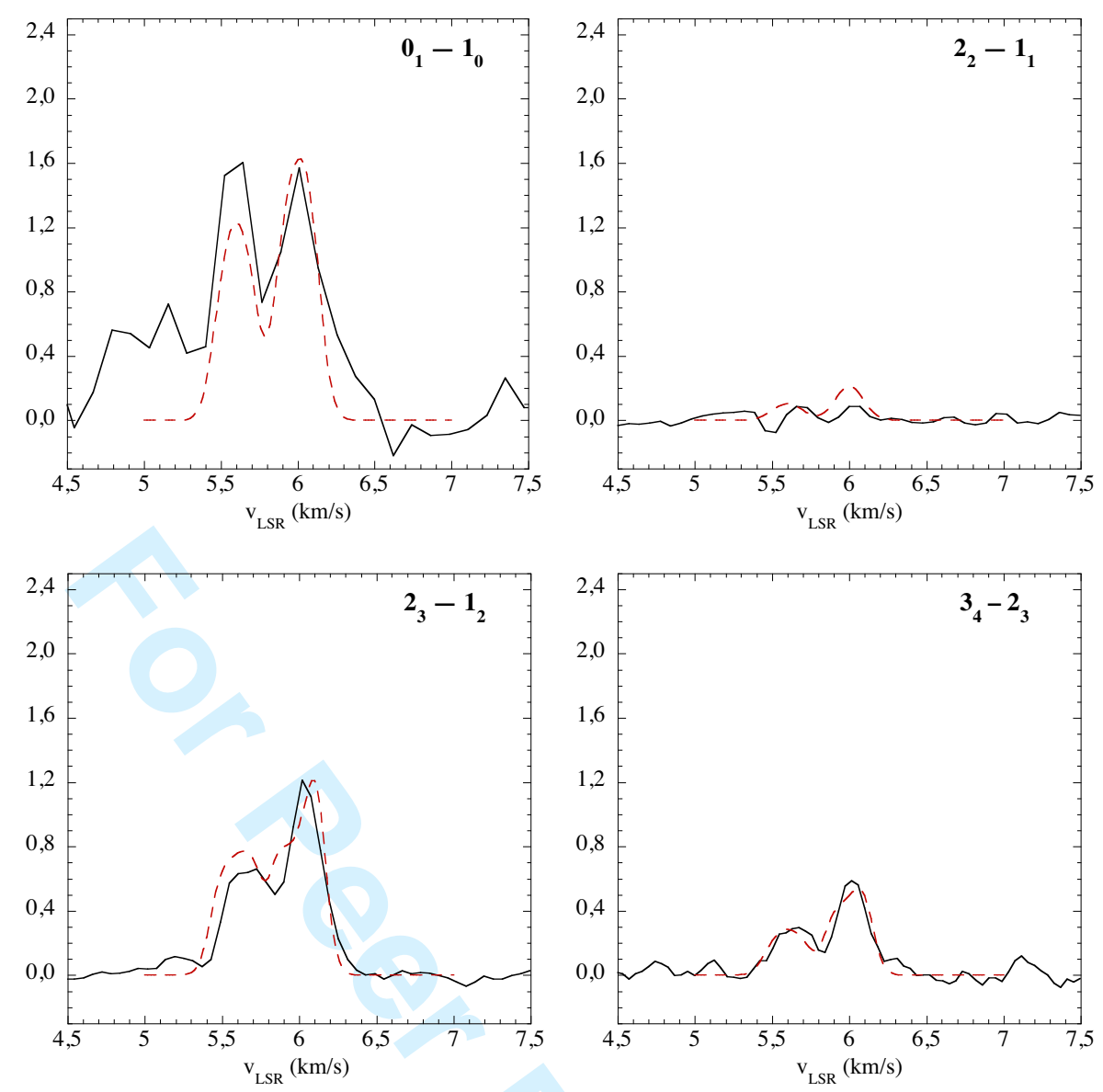

Figure 3. Theoretical line profile (dashed line) compared to SO observations in TMC-1 (full line).

to derive the physical conditions of cold molecular clouds. In particular, the use of previous rate coefficients could result in overestimating the abundance of SO by a factor 2 to 10 in cold molecular clouds.

\section{Concluding remarks}

From these few examples, it appears clearly that reliable astrophysical modeling needs accurate molecular data and in particular accurate collisional rate coefficients if the Local Thermodynamical Equilibrium is not fulfilled. This will be of a particularly great importance for the interpretation of the data that extremely powerful instruments such as Herschel and ALMA will provide in the next years.

Accurate predictions of rotational excitation cross sections can be obtained for a large set of small molecules in interaction with $\mathrm{He}$ and $\mathrm{H}_{2}$ as long as only one electronic configuration contributes to the potential energy surfaces. The accuracy of the PES can reach a few $\mathrm{cm}^{-1}$ and close-coupling calculations can be performed. This fact is actually true for low temperatures but may be more questionnable for larger temperatures. In fact, the best level of accuracy is out of reach of current approximate approaches for scattering. Accordingly, the main source of uncertainties may come in the future from the scattering calculations instead of the PES, especially for highly excited rotational states or vibrationally excited molecules, for which fully converged close-coupling calculations become very difficult.

\section{Acknowledgments}

We are very pleased to thank Pavel Rosmus for his continuous and friendly help and interest in our work. 


\section{Molecular Physics}

\section{References}

[1] F. Lique, A. Spielfiedel, J. Cernicharo. Astronomy and Astrophysics, 451, 1125 (2006).

[2] F. Lique, J. Cernicharo, P. Cox. Astrophysical Journal, 653, 1342 (2006).

[3] F. Daniel, J. Cernicharo, M.-L. Dubernet. Astrophysical Journal, 648, 461 (2006).

[4] P. F. Goldsmith. Astrophysical Journal, 176, 597 (1972).

[5] M. Wernli.PhD Thesis, University Joseph Fourier, Grenoble I, 2006.

[6] S. F. Boys, F. Bernardi. Mol. Phys., 19, 553 (1970).

[7] D. E. Woon, T. H. Dunning. J. Chem. Phys., 100, 2975 (1994).

[8] S. M. Cybulski, R. R. Toczylowski. J. Chem. Phys. 111, 10520 (1999).

[9] MOLPRO is a package of ab initio programs written by :

H.-J. Werner, and P. J. Knowles with contributions fromJ Almlöf, R. D. Amos, M. J. Deegan, S. T. Elbert, C. Hampel, W. Meyer, K. Peterson, R. Pitzer, A. J. Stone, P. R. Taylor, R. Lindh, M. E. Mura and T. Thorsteisson.

[10] A. M. Arthurs, A. Dalgarno.Proc. Roy. Soc. A, 256, 540 (1960).

[11] J. M. Hutson, S. Green. MOLSCAT computer code, version 14 (Collaborative Computational Project No. 6 of the Science and Engineering Research Council, United Kingdom, 1994).

[12] F. Dayou, C. Balança. Astronomy and Astrophysics, 459, 297 (2006).

[13] H. -J. Werner, B. Follmeg and M. H. Alexander. J. Chem. Phys. 89, 3139 (1988).

[14] C. W. McCurdy \& W. H. Miller. J. Chem. Phys. 67, 463 (1977).

[15] B. E. Turner, K. -W. Chan, S. Green and D. A. Lubowich. Astrophysical Journal, 399, 114 (1992).

[16] S. Green. Astrophysical Journal, 434, 188 (1994)

[17] D. A. Neufeld \& S. Green. Astrophysical Journal, 432, 158 (1994)

[18] F. Lique, A. Spielfiedel, M.-L. Dubernet, N. Feautrier. J. Chem. Phys., 123, 134316 (2005).

[19] V. V. Sobolev. In Theoretical Astrophysics, V. A. Ambartsumyan (Ed), chapter 29, Pergamon Press Ltd., London (1958). 


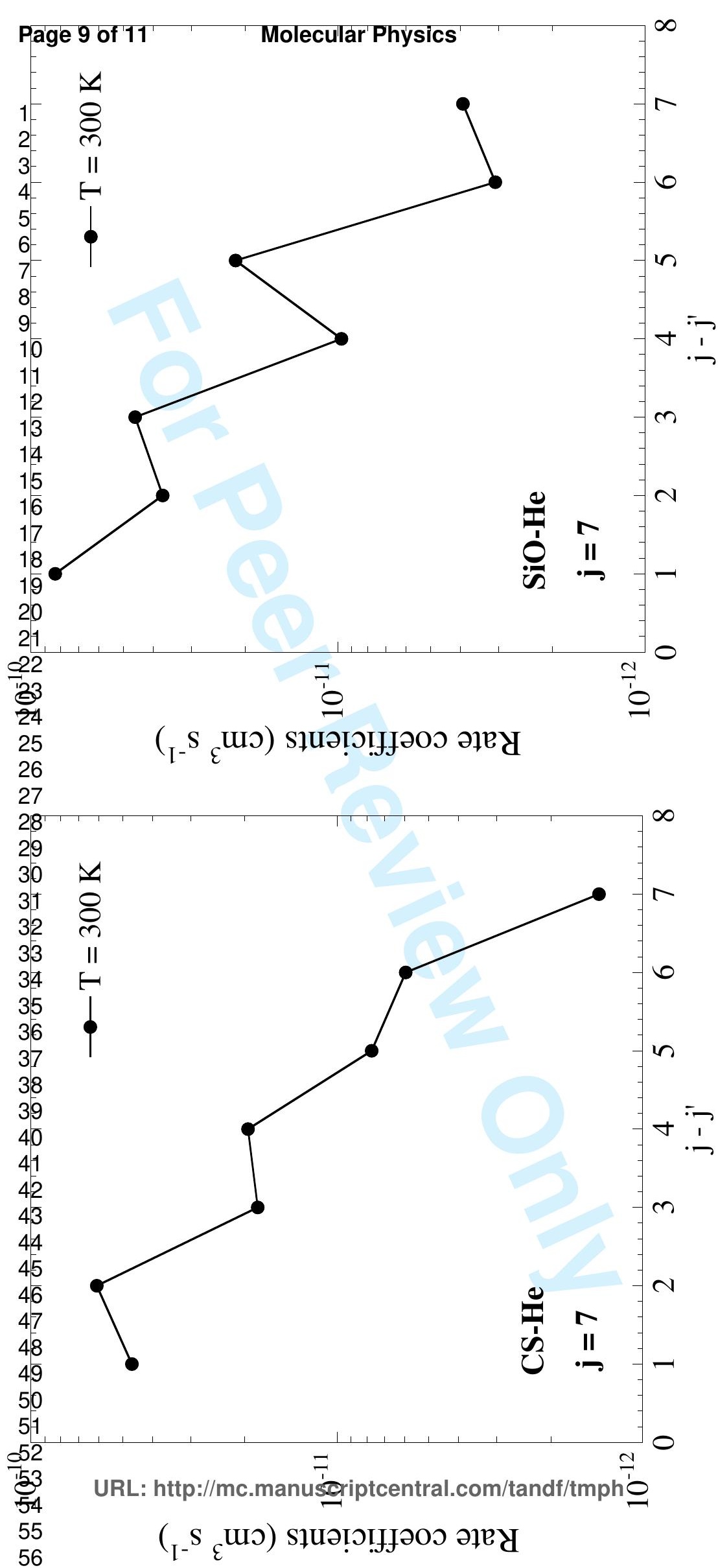




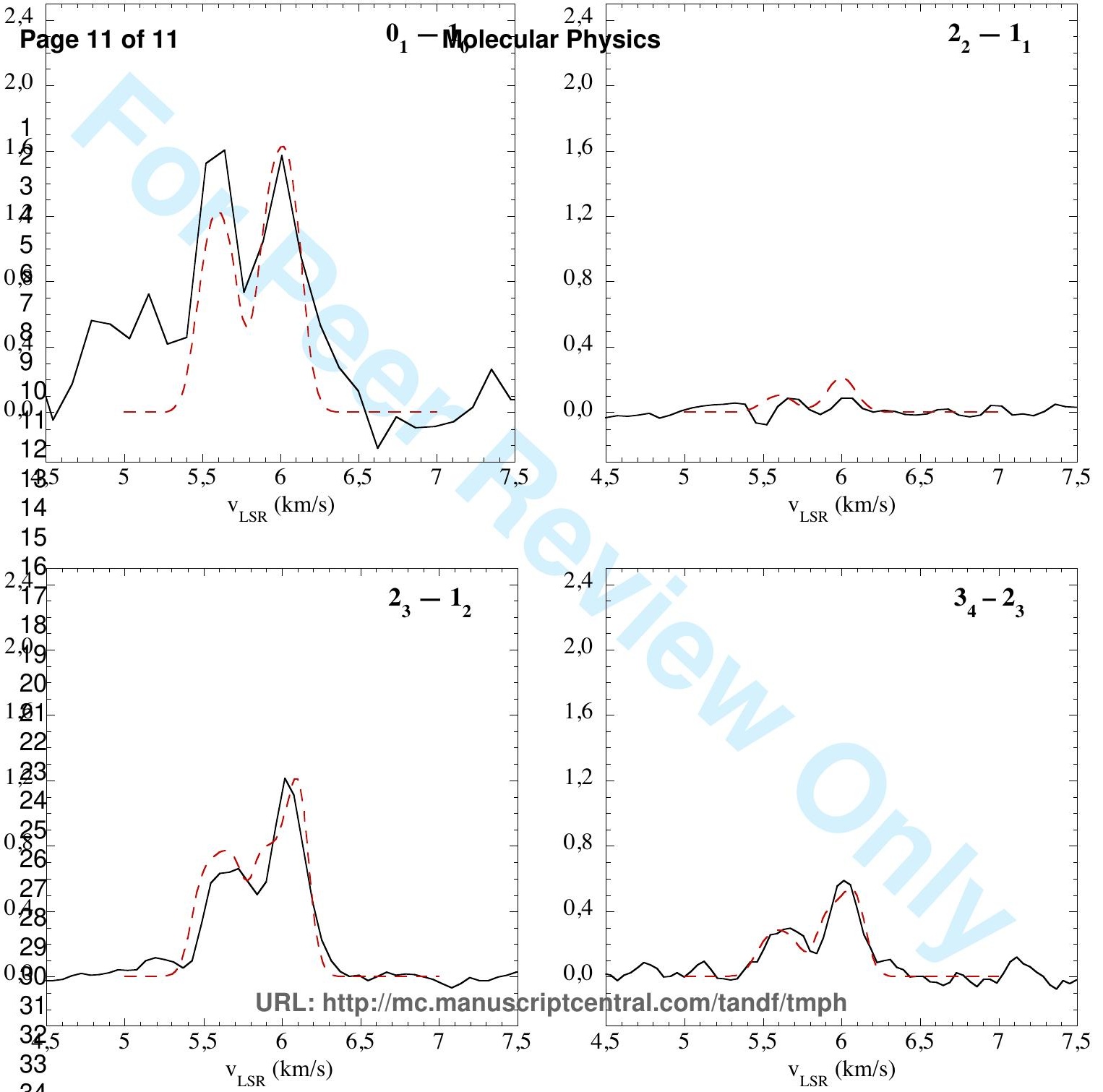

